\title{
The Erlang distribution approximates the age distribution of incidence of childhood and young adulthood cancers
}

\author{
Aleksey V Belikov ${ }^{\text {Corresp., }}{ }^{1}$, Alexey D Vyatkin ${ }^{1}$, Sergey V Leonov ${ }^{1}$ \\ ${ }^{1}$ Laboratory of Innovative Medicine, School of Biological and Medical Physics, Moscow Institute of Physics and Technology, Moscow, Russia \\ Corresponding Author: Aleksey V Belikov \\ Email address: belikov.research@gmail.com
}

Background. It is widely believed that cancers develop upon acquiring a particular number of (epi)mutations in driver genes, but the law governing the kinetics of this process is not known. We have previously shown that the age distribution of incidence for the 20 most prevalent cancers of old age is best approximated by the Erlang probability distribution. The Erlang distribution describes the probability of several successive random events occurring by the given time according to the Poisson process, which allows an estimate for the number of critical driver events.

Methods. Here we employ a computational grid search method to find global parameter optima for 5 probability distributions on the CDC WONDER dataset of the age distribution of childhood and young adulthood cancer incidence.

Results. We show that the Erlang distribution is the only classical probability distribution we found that can adequately model the age distribution of incidence for all studied childhood and young adulthood cancers, in addition to cancers of old age.

Conclusions. This suggests that the Poisson process governs driver accumulation at any age and that the Erlang distribution can be used to determine the number of driver events for any cancer type. The Poisson process implies the fundamentally random timing of driver events and their constant average rate. As waiting times for the occurrence of the required number of driver events are counted in decades, and most cells do not live this long, it suggests that driver mutations accumulate silently in the longestliving dividing cells in the body - the stem cells. 


\section{The Erlang distribution approximates the age distribution 2 of incidence of childhood and young adulthood cancers}

3 Aleksey V Belikov ${ }^{\text {Corresp, } 1}{ }^{1}$, Alexey D Vyatkin ${ }^{1}$, Sergey V Leonov ${ }^{1}$

41 Laboratory of Innovative Medicine, School of Biological and Medical Physics, Moscow Institute of Physics and Technology, Moscow, 5 Russia

6 Corresponding Author: Aleksey V Belikov Email address: belikov.research@gmail.com 
7 Background. It is widely believed that cancers develop upon acquiring a particular number of

8 (epi)mutations in driver genes, but the law governing the kinetics of this process is not known. We

9 have previously shown that the age distribution of incidence for the 20 most prevalent cancers of

10 old age is best approximated by the Erlang probability distribution. The Erlang distribution

11 describes the probability of several successive random events occurring by the given time

12 according to the Poisson process, which allows an estimate for the number of critical driver events.

13 Methods. Here we employ a computational grid search method to find global parameter optima for 145 probability distributions on the CDC WONDER dataset of the age distribution of childhood and 15 young adulthood cancer incidence.

16 Results. We show that the Erlang distribution is the only classical probability distribution we found 17 that can adequately model the age distribution of incidence for all studied childhood and young 18 adulthood cancers, in addition to cancers of old age.

Conclusions. This suggests that the Poisson process governs driver accumulation at any age and that the Erlang distribution can be used to determine the number of driver events for any cancer type.

21 The Poisson process implies the fundamentally random timing of driver events and their constant average rate. As waiting times for the occurrence of the required number of driver events are 23 counted in decades, and most cells do not live this long, it suggests that driver mutations

24 accumulate silently in the longest-living dividing cells in the body - the stem cells. 
Since the discovery of the connection between cancer and mutations in DNA, in the

27 middle of the $20^{\text {th }}$ century, there have been multiple attempts to deduce the law of driver

mutation accumulation from the age distribution of cancer incidence or mortality (Hornsby, Page \& Tomlinson, 2007). The proposed models, however, suffer from several serious drawbacks. For example, early models assume that cancer mortality increases with age according to the power law (Nordling, 1953; Armitage \& Doll, 1954; Knudson, 2001), whilst already at that time it was known that many cancers display deceleration of mortality growth at advanced age. Moreover, when large-scale incidence data have accumulated, it became clear that cancer incidence not only ceases to increase with age but, for at least some cancers, starts to decrease (Saltzstein, Behling \& Baergen, 1998; Harding, Pompei \& Wilson, 2012). More recent models of cancer progression are based on multiple unverified biological assumptions, consist of complex equations that incorporate many empirically determined parameter values, and still have not been shown to describe the decrease in cancer incidence at an advanced age (Luebeck \& Moolgavkar, 2002; Little \& Wright, 2003; Michor, Iwasa \& Nowak, 2006; Meza et al., 2008; Calabrese \& Shibata, 2010; Luebeck et al., 2013). It is also clear that an infinite number of such mechanistic models can be created and custom-tailored to fit any set of data, leading us to question their explanatory and predictive values.

Recently, we have proposed that the age distribution of cancer incidence is, in fact, a statistical distribution of probabilities for a required number of driver events to occur precisely by the given age, i.e. a probability density function (Belikov, 2017). We define driver events as discrete molecular alterations that provide the cancer cell of origin and the 
48

49

50

5

5

resulting clone the necessary means to overcome both the intracellular and extracellular tumor suppressor mechanisms, such as cell cycle checkpoints, contact inhibition, immune surveillance, and promote growth in the absence of growth factors. Thus, our concept of a driver event integrates mutational, microenvironmental, immune and evolutionary perspectives (Rozhok \& DeGregori, 2015; Solary \& Lapane, 2020). It is also important to note that only the winner clone's evolutionary history determines the time of tumour appearance and thus the kinetics of cancer incidence, therefore any mention of driver events in this article would always refer to driver events in the winner clone. The total amount of competing clones would rather affect the overall probability of having a cancer. Previously. we tested the probability density functions of 16 well-known continuous probability distributions for fits with the CDC WONDER data on the age distribution of incidence for the 20 most prevalent cancers of old age (Belikov, 2017). The best fits were observed for the gamma distribution and its special case - the Erlang distribution, with the average $\mathrm{R}^{2}$ of 0.995 (Belikov, 2017). Notably, these two distributions describe the probability of several successive independent random events occurring precisely by the given time. In fact, the Erlang distribution is the distribution of a sum of independent exponential variables, whereas the exponential distribution is the probability distribution of the time between events in a Poisson point process, i.e., a process in which events occur continuously and independently at a constant average rate (Birnbaum, 1954). This allowed us to estimate the number of driver events, the average time interval between them and the maximal populational susceptibility, for each cancer type. The results showed high heterogeneity in all three parameters amongst the cancer types but high reproducibility between the years of observation (Belikov, 2017). 
71 However, four other probability distributions - the extreme value (Gumbel), normal,

72 logistic and Weibull - also showed good fits to the data, although inferior to the gamma and

73 Erlang distributions. This leaves some uncertainty regarding the exceptionality of the 74 gamma/Erlang distribution for the description of cancer incidence. Here we test these 75 shortlisted distributions on the CDC WONDER data on childhood and young adulthood 76 cancers, using a grid search computational method to find global parameter optima. We 77 show that the gamma/Erlang distribution is the only distribution that provides close fits 78 for all tested cancers without involvement of negative age values. This result, taken 79 together with our previous findings (Belikov, 2017), suggests that driver accumulation is 80 governed by the Poisson process, both in childhood and in adult cancers. This is consistent 81 with driver mutations that accumulate randomly, silently, at constant average rate and for 82 many decades, likely in stem cells. 
83

84

85

86

87

88

\section{METHODS}

\section{Data acquisition}

Data were collected as previously described in (Belikov, 2017). Briefly, United States Cancer Statistics Public Information Data: Incidence 1999 - 2012 were downloaded via CDC WONDER online database (http://wonder.cdc.gov/cancer-v2012.HTML). Results were grouped by 5-year Age Groups, Crude Rates were selected as an output and All Ages were selected in the Age Group box. The data were downloaded separately for each specific cancer type, upon its selection in the Childhood Cancers tab. Only cancers that show childhood/young adulthood incidence peaks and do not show middle/old age incidence peaks were analysed further. The middle age of each age group was used as the x value, e.g. 17.5 for the "15-19 years" age group.

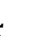

\section{Data analysis}

For data analysis, custom scripts were written in Python 3.7, now publicly available at our GitHub repository: https://github.com/belikov-av/childhoodcancers The following packages were used: Scikit-learn 0.22.1, Numpy 1.18.1, Scipy 1.4.1, Pandas 1.0.3, Plotly 4.5.4 and Matplotlib 3.1.3. Distributions were defined as shown in Table 1.

In order to find and visually demonstrate the globally optimal parameters for every distribution, a grid search method (Jiménez, Lázaro \& Dorronsoro, 2007) was chosen, as opposed to standard maximum likelihood estimation methods that do not guarantee finding the global optimum. Grid search allowed us to explore all combinations of values for two parameters $(k$ and $b$ for the Erlang and Weibull distributions; $\mu$ and $b$ for the extreme value, logistic and normal distributions) within the defined limits and with the defined 
106 steps (400 values for each parameter, 160000 grid nodes). For each pair of parameter 107 values, the optimum value for the third parameter (amplitude, interpreted as the maximal 108 populational susceptibility, see Belikov, 2017) was found using the golden section search 109 method (Pejic \& Arsic, 2019). In order to perform the golden section search, first the area 110 under the incidence curve was estimated as the sum of actual incidence values for each age 111 group. The left and right boundaries for the golden section search were then defined as an 112 estimated area under the curve divided or multiplied by 100, respectively. After the 113 optimal amplitude parameter value was found with the golden section search, the fitted

114 distribution was generated and plotted alongside the incidence data and the goodness of fit $115\left(R^{2}\right)$ was calculated, for each node of the grid. Finally, the node with the highest $R^{2}$ was 116 selected and its parameters deemed the global optimum. 


\section{$117 \quad$ RESULTS}

118 To test the universality and exceptionality of the gamma/Erlang distribution, the

119 publicly available USA incidence data on childhood and young adulthood cancers were

120 downloaded from the CDC WONDER database (see Methods). In addition to the

121 gamma/Erlang distribution, the probability density functions of the following continuous

122 probability distributions were selected for testing based on their good fits to adult cancers

123 (Belikov, 2017): extreme value (Gumbel), logistic, normal and Weibull. We used a custom

124 grid search script to explore all combinations of parameters and find global $\mathrm{R}^{2}$ maxima (see

125 Figure 1 for gamma/Erlang distribution, Supplemental Material for other distributions and

126 Methods for the detailed description). Results showed that the extreme value (Gumbel),

127 logistic and normal distributions fit to some childhood cancers in a way that a large part of

128 their probability density function appears in the negative range of $\mathrm{x}$ values (Figure 2). As

129 the $\mathrm{x}$ axis corresponds to patients' ages, this makes these three distributions

130 uninterpretable in the biological context. Hence, they were excluded from the further

131 analysis. The remaining gamma/Erlang and Weibull distributions are defined only for

132 positive $\mathrm{x}$ values, so there was no such problem (Figure 2). The gamma/Erlang distribution

133 provided closer fits to the childhood and young adulthood cancers than the Weibull

134 distribution (Figure 3, Table 2).

135 Importantly, the gamma distribution and the Erlang distribution derived from it are

136 the only classical continuous probability distributions that describe the cumulative waiting

137 time for $k$ successive random events, with the Erlang distribution differing only in counting

138 events as integer numbers. Assuming this model describes the waiting time for real

139 discrete random events such as driver mutations, the gamma/Erlang distribution provides 
140 the opportunity to get unique insights into the carcinogenesis process. We have previously

141 proposed that the shape parameter $k$ of the gamma/Erlang distribution indicates the

142 average number of driver events that need to occur in order for a cancer to develop to a

143 stage that can be detected during clinical screening; the scale parameter $b$ indicates the

144 average time interval (in years) between such events; and the amplitude parameter $A$

145 divided by 1000 estimates the maximal susceptibility (in percent) of a given population to a

146 given type of cancer (Belikov, 2017). 
147 To obtain these parameter values, the gamma/Erlang distribution was fitted 148 individually to incidence of each of 10 childhood/young adulthood cancer types (Figure 3, 149 Table 3).

150 The non-integer values of the shape parameter $k$ can be easily explained if we suppose 151 that the studied population consists of the mixture of patients with slightly different 152 numbers of driver events. The goodness of fit varied from 0.9495 , for extracranial and 153 extragonadal germ cell tumours of young adulthood, to 1.000, for extracranial and 154 extragonadal germ cell tumours of childhood and retinoblastoma, with the average of 155 0.9854. The predicted number of driver events varied from 0.4, for extracranial and 156 extragonadal germ cell tumours of childhood, to 8.95, for malignant gonadal germ cell 157 tumours. The predicted average time between the events varied from 1.4 years, for 158 hepatoblastoma, to 14.85 years, for intracranial and intraspinal embryonal tumours. The 159 predicted maximal populational susceptibility varied from $0.0322 \%$, for extracranial and 160 extragonadal germ cell tumours of childhood, to $1.966 \%$, for malignant gonadal germ cell 161 tumours. Overall, the data show the same high heterogeneity in carcinogenesis patterns 162 revealed in the previous study (Belikov, 2017). 
163

164

165

166 (Gumbel), gamma/Erlang, normal, logistic and Weibull - approximate the age distribution

167 of incidence for the 20 most prevalent cancers of old age (Belikov, 2017). The shape of

168 those incidence distributions resembles the bell shape of the normal distribution, with 169 170

171

172

173

174 175 176 177 178 179 180 181 182 183 184 185 186 some asymmetry, or at least the left part of it. However, many cancers of childhood have a radically different shape of the incidence distribution, resembling the shape of the exponential distribution (Figure 3). Of the five shortlisted distributions, only the gamma/Erlang and Weibull distributions can assume such shape, when the parameter $k$ equals one or less. Other distributions can fit the data only by extending their probability density function into the negative range of age values (Figure 2), which makes their proper biological interpretation highly unlikely. Of the remaining two distributions, the gamma/Erlang provides superior fit compared to Weibull. In fact, for cancers of old age, the average $\mathrm{R}^{2}$ for the Weibull distribution is 0.9938 , whereas for the gamma/Erlang distribution is 0.9954 (Belikov, 2017). For cancers of childhood and young adulthood, the average $\mathrm{R}^{2}$ for the Weibull distribution is 0.9707 , whereas for the gamma/Erlang distribution is 0.9854 (Table 2). Thus, it appears that the gamma/Erlang distribution is the only classical probability distribution that fits universally well to cancers of childhood, young adulthood and old age. The Weibull distribution can also approximate cancer incidence (Webster, 2019), but would only be exactly correct for cancers with $k=1$, when it becomes the exponential distribution.

We have proposed that the parameter $k$ of the Erlang distribution indicates the average number of driver events that need to occur in order for a cancer to develop to a 
187 stage that can be detected during clinical screening (Belikov, 2017). The Erlang

188 distribution, like the Weibull distribution, reduces to the exponential distribution when $k$

189 equals one, because the exponential distribution describes the waiting time for a single

190 random event. It would thus mean that cancers with the exponential shape of the age

191 distribution of incidence require only a single driver event with random time of

192 occurrence, most likely a somatic driver mutation (Pon \& Marra, 2015) or epimutation

193 (Roy, Walsh \& Chan, 2014). This explains their maximal prevalence in the early childhood.

194 In his seminal paper (Knudson, 1971), Alfred Knudson has proposed that hereditary

195 retinoblastoma is caused by a single somatic mutation in addition to one heritable

196 mutation. He also proposed that in the nonhereditary form of the disease, both mutations

197 should occur in somatic cells. As hereditary form is estimated to represent about $45 \%$ of all

198 cases (Knudson, 1971; Dimaras et al., 2012), the number of driver mutations predicted

199 from combined incidence data should be around 1.55. Interestingly, whilst the 200 gamma/Erlang distribution fits the incidence data excellently, with $\mathrm{R}^{2}=1.0$, it predicts 1.3

201 driver events (Table 3). This yields the estimate of the hereditary form prevalence at $70 \%$.

202 This higher value may point to the general underestimation of the hereditary component in 203 unilateral retinoblastoma, perhaps due to limitations of routine genetic screening and the 204 influence of genetic mosaicism (Chen et al., 2014). In contrast to retinoblastoma, the 205 hereditary form of neuroblastoma is estimated to comprise only 1-2\% of all cases (Tolbert 206 et al., 2017), hence the exponential age distribution of incidence would mean that only one 207 somatic mutation is required. Indeed, the gamma/Erlang distribution predicts one driver 208 event (Table 3). 
210 of incidence does not mean that only a single driver gene can be discovered in such cancer

211 types. In fact, many driver genes are redundant or even mutually exclusive, e.g. when the

212 corresponding proteins are components of the same signalling pathway (Vandin, Upfal \&

213 Raphael, 2012). Thus, each tumour in such cancer types is expected to have a mutation in

214 one driver gene out of a set of several possible ones, in which all genes most likely encode

215 members of the same pathway or are responsible for the same cellular function. For

216 example, in each neuroblastoma tumour sample, a mutation was present in only one out of

217 five putative driver genes - ALK, ATRX, PTPN11, MYCN or NRAS (Pugh et al., 2013).

218 Another aspect to consider is that while one mutation is usually sufficient to activate an

219 oncogene, two mutations are typically required to inactivate both alleles of a tumour

220 suppressor gene. Therefore, cancers with the exponential age distribution of incidence are

221 predicted to have either a single somatic mutation in an oncogene, or a single somatic

222 mutation in a tumour suppressor gene plus an inherited mutation in the same gene. The

223 former is the case for neuroblastoma, where an amplification or an activating point

224 mutation in $A L K$ is often present (Chen et al., 2008; George et al., 2008; Janoueix-Lerosey et

225 al., 2008). The latter is the case for retinoblastoma, where an inactivating mutation in one

226 allele of $R B 1$ is usually inherited, whereas an inactivating mutation in the other $R B 1$ allele

227 occurs in a somatic cell (Friend et al., 1986).

228 The number of driver events predicted by the Erlang distribution refers exclusively to

229 rate-limiting events responsible for cancer progression. For example, it was shown that

230 inactivation of both alleles of $R B 1$ leads first to retinoma, a benign tumour with genomic

231 instability that easily transforms to retinoblastoma upon acquiring additional mutations 
232 (Dimaras et al., 2008). In this case, two mutations in $R B 1$ are rate-limiting, whereas

233 mutations in other genes are not, because genomic instability allows them to occur very 234 quickly. In neuroblastoma, frequent $M Y C N$ amplification and chromosome 17q gain are 235 found only in advanced stages of the disease (Brodeur et al., 1984; Bown et al., 1999), so 236 they are unlikely to be the initiating rate-limiting events.

237 Application of the gamma/Erlang distribution to childhood and young adulthood 238 cancers showed its exceptionality amongst other probability distributions. The fact that it 239 can successfully describe the radically different age distributions of incidence for cancers of 240 any age and any type suggests that the underlying Poisson process is the universal 241 principle governing cancer development. The Poisson process implies the fundamentally 242 random timing of driver events and their constant average rate (Belikov, 2017). Indeed, it 243 has been shown that the number of mutations in cancers (Tomasetti, Vogelstein \& 244 Parmigiani, 2013) and stem cells (Blokzijl et al., 2016) from various tissues increases 245 linearly with age, i.e. with constant rates. The gamma/Erlang distribution allows to 246 estimate, by multiplying the number of driver events by the average time interval between 247 them, that an average person needs from 73 to 324 years to accumulate the required 248 number of driver alterations, depending on the cancer type (Belikov, 2017). These 249 estimates correspond to peaks in cancer incidence, because accumulating mutations faster 250 or slower than at an average rate is less likely. Notably, only the susceptible part of the 251 population will develop a given type of cancer, hence the area under the incidence curve 252 never reaches the total population size. Moreover, the finding that driver mutations 253 accumulate for decades is consistent with the silent accumulation of driver mutations in 254 stem cells before the terminal clonal expansion (Tomasetti \& Vogelstein, 2015; Blokzijl et 
255 al., 2016; Gerstung et al., 2020), because this is the only type of dividing cells surviving for

256 so long in the body, and DNA damage requires cellular division to be fixed in the form of

257 mutations. For childhood and young adulthood cancers, these estimates range from one to

25835 years (see Table 3), but the mechanism is likely the same.

259 Intriguingly, the parameters $k$ (the number of driver events) and $b$ (the average 260 interval between them, in years) demonstrate inverse correlation in adult cancers (see 261 Table 1 in Belikov, 2017). This could be explained by the observation that all incidence 262 peaks of adult cancers are located at the age of 73 years old or greater, therefore cancer 263 types with high mutation rates (and thus low $b$ values) would accumulate more driver 264 mutations $(k)$ by that time than cancer types with low mutation rates (and thus high $b$ 265 values). Although this answers the initial question it raises a new one: why cancers with 266 high mutation rates do not simply peak at earlier ages and with lower $k$, if such low $k$ is 267 sufficient for low mutation rate cancers? One likely explanation is that an evolutionary 268 pressure of natural selection ensured that cancers do not routinely appear during the 269 reproductive period of life and therefore increased the number of tumour suppressor 270 mechanisms to overcome by high mutation rate cancers. Thus, common cancers can appear 271 only at the old age when evolutionary pressure is absent or minimal. Recently. it has been 272 proposed that age-related degradation of stem cell niche microenvironment is the major 273 reason for positive selection of driver mutations in stem cells and for late-life cancer 274 incidence peaks (Rozhok \& DeGregori, 2015; Rozhok \& De Gregori, 2019). That theory, 275 however, has yet to explain the decline in incidence after the peak.

276 Simulations suggest that the number of human (hematopoietic) stem cells increases 277 from birth until adolescence and then plateaus (Catlin et al., 2011). This should lead to the 
278 gradual increase in childhood cancer incidence with age, as the target population for

279 mutations grows. On the other hand, modelling suggests that the replication rate of human

280 (hematopoietic) stem cells decreases from $\sim 17$ times in the first year of life, to $\sim 2.5$

281 times/year between the ages of 3 and 13 years, to $~ 0.6$ times/year in adults (Sidorov et al.,

282 2009). This should lead to gradual decrease in childhood cancer incidence with age, as the

283 stem cell mutation rate heavily depends on the cell division rate. What is the net effect of

284 these two processes with opposing effects on childhood cancer incidence is not clear

285 (Rozhok \& DeGregori, 2015). Our modelling with the constant driver event rate parameter

286 closely approximates the actual incidence of multiple childhood cancers, which suggests

287 that increases in stem cell pool sizes and decreases in stem cell division rates indeed

288 counterbalance each other and have negligible net effect on childhood cancer incidence.

289 Another interesting prediction of our model would be that the number of driver 290 mutations per stem cell or per tumour would have a Poisson distribution across the 291 patients' population of any given age. Finally, as the gamma/Erlang distribution allows to 292 predict the number and rate of driver events in any cancer subtype for which the data on 293 the age distribution of incidence are available, it may help to optimize the algorithms for 294 distinguishing between driver and passenger mutations (Raphael et al., 2014), leading to 295 the development of more effective targeted therapies.

\section{Acknowledgements}

AVB acknowledges MIPT 5-100 program support for early career researchers. 


\section{References}

301 Armitage P, Doll R. 1954. The age distribution of cancer and a multi-stage theory of 302 carcinogenesis. British Journal of Cancer 8. DOI: 10.1038/bjc.1954.1.

303 Belikov AV. 2017. The number of key carcinogenic events can be predicted from cancer 304 incidence. Scientific Reports 7. DOI: 10.1038/s41598-017-12448-7.

305 Birnbaum A. 1954. Statistical Methods for Poisson Processes and Exponential

306 Populations. Journal of the American Statistical Association 49. DOI:

307 10.1080/01621459.1954.10483504.

308 Blokzijl F, De Ligt J, Jager M, Sasselli V, Roerink S, Sasaki N, Huch M, Boymans S, Kuijk E, 309 Prins P, Nijman IJ, Martincorena I, Mokry M, Wiegerinck CL, Middendorp S, Sato T, Schwank 310 G, Nieuwenhuis EES, Verstegen MMA, Van Der Laan LJW, De Jonge J, Ijzermans JNM, Vries

311 RG, Van De Wetering M, Stratton MR, Clevers H, Cuppen E, Van Boxtel R. 2016. Tissue312 specific mutation accumulation in human adult stem cells during life. Nature 538. DOI: 313 10.1038/nature19768.

314 Bown N, Cotterill S, Łastowska M, O’Neill S, Pearson ADJ, Plantaz D, Meddeb M, Danglot 315 G, Brinkschmidt C, Christiansen H, Laureys G, Nicholson J, Bernheim A, Betts DR, 316 Vandesompele J, Van Roy N, Speleman F. 1999. Gain of Chromosome Arm 17q and Adverse 317 Outcome in Patients with Neuroblastoma. New England Journal of Medicine 340. DOI: 318 10.1056/nejm199906243402504.

319 Brodeur GM, Seeger RC, Schwab M, Varmus HE, Michael Bishop J. 1984. Amplification of $320 \mathrm{~N}$-myc in untreated human neuroblastomas correlates with advanced disease stage. Science 321 224. DOI: 10.1126/science.6719137.

322 Calabrese P, Shibata D. 2010. A simple algebraic cancer equation: Calculating how 323 cancers may arise with normal mutation rates. BMC Cancer 10. DOI: 10.1186/1471-2407$324 \quad 10-3$.

325 Catlin SN, Busque L, Gale RE, Guttorp P, Abkowitz JL. 2011. The replication rate of 326 human hematopoietic stem cells in vivo. Blood 117:4460-4466. DOI: 10.1182/blood-2010327 08-303537.

328 Chen Z, Moran K, Richards-Yutz J, Toorens E, Gerhart D, Ganguly T, Shields CL, Ganguly 329 A. 2014. Enhanced sensitivity for detection of low-level germline mosaic RB1 mutations in 
330 sporadic retinoblastoma cases using deep semiconductor sequencing. Human Mutation 35.

331 DOI: $10.1002 /$ humu.22488.

332 Chen Y, Takita J, Choi YL, Kato M, Ohira M, Sanada M, Wang L, Soda M, Kikuchi A, Igarashi

333 T, Nakagawara A, Hayashi Y, Mano H, Ogawa S. 2008. Oncogenic mutations of ALK kinase in 334 neuroblastoma. Nature 455. DOI: 10.1038/nature07399.

335 Dimaras H, Khetan V, Halliday W, Orlic M, Prigoda NL, Piovesan B, Marrano P, Corson 336 TW, Eagle RC, Squire JA, Gallie BL. 2008. Loss of RB1 induces non-proliferative retinoma: 337 Increasing genomic instability correlates with progression to retinoblastoma. Human 338 Molecular Genetics 17. DOI: 10.1093/hmg/ddn024.

339 Dimaras H, Kimani K, Dimba EAO, Gronsdahl P, White A, Chan HSL, Gallie BL. 2012.

340 Retinoblastoma. DOI: 10.1016/S0140-6736(11)61137-9.

341 Friend SH, Bernards R, Rogelj S, Weinberg RA, Rapaport JM, Albert DM, Dryja TP. 1986. A 342 human DNA segment with properties of the gene that predisposes to retinoblastoma and 343 osteosarcoma. Nature 323. DOI: 10.1038/323643a0.

344 George RE, Sanda T, Hanna M, Fröhling S, Luther W, Zhang J, Ahn Y, Zhou W, London WB, 345 McGrady P, Xue L, Zozulya S, Gregor VE, Webb TR, Gray NS, Gilliland DG, Diller L, Greulich 346 H, Morris SW, Meyerson M, Look AT. 2008. Activating mutations in ALK provide a 347 therapeutic target in neuroblastoma. Nature 455. DOI: 10.1038/nature07397.

348 Gerstung M, Jolly C, Leshchiner I, Dentro SC, Gonzalez S, Rosebrock D, Mitchell TJ, 349 Rubanova Y, Anur P, Yu K, Tarabichi M, Deshwar A, Wintersinger J, Kleinheinz K, Vázquez350 García I, Haase K, Jerman L, Sengupta S, Macintyre G, Malikic S, Donmez N, Livitz DG, Cmero 351 M, Demeulemeester J, Schumacher S, Fan Y, Yao X, Lee J, Schlesner M, Boutros PC, Bowtell 352 DD, Zhu H, Getz G, Imielinski M, Beroukhim R, Sahinalp SC, Ji Y, Peifer M, Markowetz F, 353 Mustonen V, Yuan K, Wang W, Morris QD, Yu K, Adams DJ, Campbell PJ, Cao S, Christie EL, 354 Cun Y, Dawson KJ, Drews RM, Eils R, Fittall M, Garsed DW, Ha G, Lee-Six H, Martincorena I, 355 Oesper L, Peto M, Raphael BJ, Salcedo A, Shi R, Shin SJ, Spiro O, Stein LD, Vembu S, Wheeler 356 DA, Yang TP, Spellman PT, Wedge DC, Van Loo P. 2020. The evolutionary history of 2,658 357 cancers. Nature 578. DOI: 10.1038/s41586-019-1907-7.

358 Harding C, Pompei F, Wilson R. 2012. Peak and decline in cancer incidence, mortality, 359 and prevalence at old ages. Cancer 118. DOI: 10.1002/cncr.26376.

360 Hornsby C, Page KM, Tomlinson IP. 2007. What can we learn from the population 
361 incidence of cancer? Armitage and Doll revisited. Lancet Oncology 8. DOI: 10.1016/S1470362 2045(07)70343-1.

363 Janoueix-Lerosey I, Lequin D, Brugières L, Ribeiro A, De Pontual L, Combaret V, Raynal V, 364 Puisieux A, Schleiermacher G, Pierron G, Valteau-Couanet D, Frebourg T, Michon J, Lyonnet 365 S, Amiel J, Delattre 0. 2008. Somatic and germline activating mutations of the ALK kinase 366 receptor in neuroblastoma. Nature 455. DOI: 10.1038/nature07398.

367 Jiménez ÁB, Lázaro JL, Dorronsoro JR. 2007. Finding Optimal Model Parameters by 368 Discrete Grid Search. In: Corchado E, Corchado JM, Abraham A eds. Innovations in Hybrid 369 Intelligent Systems. Advances in Soft Computing. Berlin, Heidelberg: Springer Berlin 370 Heidelberg, 120-127. DOI: 10.1007/978-3-540-74972-1_17.

371 Knudson AG. 1971. Mutation and cancer: statistical study of retinoblastoma. Proceedings 372 of the National Academy of Sciences of the United States of America 68. DOI:

373 10.1073/pnas.68.4.820.

374 Knudson AG. 2001. Two genetic hits (more or less) to cancer. Nature Reviews Cancer 1. 375 DOI: $10.1038 / 35101031$.

376 Little MP, Wright EG. 2003. A stochastic carcinogenesis model incorporating genomic 377 instability fitted to colon cancer data. Mathematical Biosciences 183. DOI: 10.1016/S0025378 5564(03)00040-3.

379 Luebeck EG, Curtius K, Jeon J, Hazelton WD. 2013. Impact of tumor progression on 380 cancer incidence curves. Cancer Research 73. DOI: 10.1158/0008-5472.CAN-12-2198.

Luebeck EG, Moolgavkar SH. 2002. Multistage carcinogenesis and the incidence of 382 colorectal cancer. Proceedings of the National Academy of Sciences of the United States of 383 America 99. DOI: 10.1073/pnas.222118199.

384 Meza R, Jeon J, Moolgavkar SH, Georg Luebeck E. 2008. Age-specific incidence of cancer: 385 Phases, transitions, and biological implications. Proceedings of the National Academy of 386 Sciences of the United States of America 105. DOI: 10.1073/pnas.0801151105.

387 Michor F, Iwasa Y, Nowak MA. 2006. The age incidence of chronic myeloid leukemia can 388 be explained by a one-mutation model. Proceedings of the National Academy of Sciences of 389 the United States of America 103. DOI: 10.1073/pnas.0607006103.

390 Nordling CO. 1953. A new theory on the cancer-inducing mechanism. British Journal of 391 Cancer 7. DOI: 10.1038/bjc.1953.8. 
Pejic D, Arsic M. 2019. Minimization and Maximization of Functions: Golden-Section

393 Search in One Dimension. In: Milutinovic V, Kotlar M eds. Exploring the DataFlow

394 Supercomputing Paradigm. Computer Communications and Networks. Cham: Springer

395 International Publishing, 55-90. DOI: 10.1007/978-3-030-13803-5_3.

396 Pon JR, Marra MA. 2015. Driver and Passenger Mutations in Cancer. Annual Review of

397 Pathology: Mechanisms of Disease 10:25-50. DOI: 10.1146/annurev-pathol-012414-

398040312.

399 Pugh TJ, Morozova O, Attiyeh EF, Asgharzadeh S, Wei JS, Auclair D, Carter SL, Cibulskis K, 400 Hanna M, Kiezun A, Kim J, Lawrence MS, Lichenstein L, Mckenna A, Pedamallu CS, Ramos 401 AH, Shefler E, Sivachenko A, Sougnez C, Stewart C, Ally A, Birol I, Chiu R, Corbett RD, Hirst 402 M, Jackman SD, Kamoh B, Khodabakshi AH, Krzywinski M, Lo A, Moore RA, Mungall KL, 403 Qian J, Tam A, Thiessen N, Zhao Y, Cole KA, Diamond M, Diskin SJ, Mosse YP, Wood AC, Ji L, 404 Sposto R, Badgett T, London WB, Moyer Y, Gastier-Foster JM, Smith MA, Auvil JMG, Gerhard 405 DS, Hogarty MD, Jones SJM, Lander ES, Gabriel SB, Getz G, Seeger RC, Khan J, Marra MA, 406 Meyerson M, Maris JM. 2013. The genetic landscape of high-risk neuroblastoma. Nature 407 Genetics 45. DOI: 10.1038/ng.2529.

408 Raphael BJ, Dobson JR, Oesper L, Vandin F. 2014. Identifying driver mutations in 409 sequenced cancer genomes: Computational approaches to enable precision medicine. 410 Genome Medicine 6. DOI: 10.1186/gm524.

411 Roy DM, Walsh LA, Chan TA. 2014. Driver mutations of cancer epigenomes. Protein and 412 Cell 5. DOI: 10.1007/s13238-014-0031-6.

413 Rozhok A, De Gregori J. 2019. A generalized theory of age-dependent carcinogenesis. 414 elife 8. DOI: 10.7554/eLife.39950.

415 Rozhok AI, DeGregori J. 2015. Toward an evolutionary model of cancer: Considering the 416 mechanisms that govern the fate of somatic mutations. Proceedings of the National 417 Academy of Sciences 112:8914. DOI: 10.1073/pnas.1501713112.

418 Saltzstein SL, Behling CA, Baergen RN. 1998. Features of cancer in nonagenarians and 419 centenarians. Journal of the American Geriatrics Society 46. DOI: 10.1111/j.1532420 5415.1998.tb02755.x.

421 Sidorov I, Kimura M, Yashin A, Aviv A. 2009. Leukocyte telomere dynamics and human 422 hematopoietic stem cell kinetics during somatic growth. Experimental Hematology 37:514- 
423 524. DOI: 10.1016/j.exphem.2008.11.009.

424 Solary E, Lapane L. 2020. The role of host environment in cancer evolution. Evolutionary 425 Applications 13. DOI: 10.1111/eva.13039.

426 Tolbert VP, Coggins GE, Maris JM, Maris JM. 2017. Genetic susceptibility to

427 neuroblastoma. Current Opinion in Genetics and Development 42. DOI:

428 10.1016/j.gde.2017.03.008.

429 Tomasetti C, Vogelstein B. 2015. Variation in cancer risk among tissues can be explained 430 by the number of stem cell divisions. Science 347:78-81. DOI: 10.1126/science.1260825.

431 Tomasetti C, Vogelstein B, Parmigiani G. 2013. Half or more of the somatic mutations in 432 cancers of self-renewing tissues originate prior to tumor initiation. Proceedings of the 433 National Academy of Sciences of the United States of America 110. DOI:

$434 \quad 10.1073 /$ pnas.1221068110.

435 Vandin F, Upfal E, Raphael BJ. 2012. De novo discovery of mutated driver pathways in 436 cancer. Genome Research 22. DOI: 10.1101/gr.120477.111.

437 Webster AJ. 2019. Multi-stage models for the failure of complex systems, cascading 438 disasters, and the onset of disease. PLOS ONE 14:e0216422. DOI:

439 10.1371/journal.pone.0216422.

440 


\section{Table $\mathbf{1}$ (on next page)}

Probability density functions and Python code for statistical distributions 


\begin{tabular}{|c|c|c|}
\hline Distribution & $\begin{array}{l}\text { Probability density } \\
\text { function }\end{array}$ & Python code \\
\hline Gamma/Erlang & $f(t)=\frac{1}{b \Gamma(k)}\left(\frac{t}{b}\right) k-1 e^{-\frac{t}{b}}$ & $\begin{array}{l}\text { def Erlang_pdf(k, b, t): } \\
\text { return }\left(t^{* *}(k-1) * n p \cdot \exp (-t / \text { float }(b))\right) / \\
\left(b^{* *} k * \text { gamma }(k)\right)\end{array}$ \\
\hline Weibull & $f(t)=\frac{k}{b}\left(\frac{t}{b}\right) k-1 e^{-\left(\frac{t}{b}\right) k}$ & $\begin{array}{l}\text { def Weibull_pdf(k, b, t): } \\
\quad \text { return }(k / b)^{*}(t / b)^{* *(k-1) * n p . e x p(-~} \\
(t / b)^{* * k)}\end{array}$ \\
\hline Extreme value & $f(t)=\frac{1}{b} e^{-\left(\frac{t-\mu}{b}\right)} e^{-e^{-\left(\frac{t-\mu}{b}\right)}}$ & $\begin{array}{l}\text { def Extreme_value_pdf(mu, b,t): } \\
\text { return np.exp }((m u-t) / b)^{*}(1 / b)^{*} \\
n p \cdot \exp (-n p \cdot \exp ((m u-t) / b))\end{array}$ \\
\hline Logistic & $f(t)=\frac{e^{\left(\frac{t-\mu}{b}\right)}}{b\left(1+e^{\left(\frac{t-\mu}{b}\right)}\right) 2}$ & $\begin{array}{l}\text { def Logistic_pdf(mu, b, t): } \\
\text { return }(1 / \mathrm{b}) * n p \cdot \exp ((t-m u) / b) / \\
n p . \operatorname{square}(1+n p \cdot \exp ((t-m u) / b))\end{array}$ \\
\hline Normal & $f(t)=\frac{1}{b \sqrt{2 \pi}} e^{-\frac{1}{2}\left(\frac{t-\mu}{b}\right)^{2}}$ & $\begin{array}{l}\text { def Normal_pdf(mu, b, t): } \\
\text { return }(1 /(b * n p . s q r t(2 * \text { pi }))) * n p . \exp (-0.5 \\
* \text { np.square }((t-m u) / b))\end{array}$ \\
\hline
\end{tabular}

1 


\section{Table 2 (on next page)}

Comparison of the goodness of fit $\left(R^{2}\right)$ of the gamma/Erlang and Weibull distributions to the actual age distributions of childhood/young adulthood cancer incidence.

The best fit for each cancer type is highlighted in bold. See Figure 3 for graphical representation. 
1

\begin{tabular}{|l|l|l|}
\hline Cancer type & gamma/Erlang & Weibull \\
\hline Ewing tumour and related sarcomas of bone & $\mathbf{0 . 9 5 1 6}$ & 0.9476 \\
\hline $\begin{array}{l}\text { Extracranial and extragonadal germ cell tumours of } \\
\text { childhood }\end{array}$ & $\mathbf{1 . 0 0 0}$ & $\mathbf{1 . 0 0 0}$ \\
\hline $\begin{array}{l}\text { Extracranial and extragonadal germ cell tumours of young } \\
\text { adulthood }\end{array}$ & $\mathbf{0 . 9 4 9 5}$ & 0.8689 \\
\hline Hepatoblastoma & $\mathbf{0 . 9 9 9 6}$ & $\mathbf{0 . 9 9 9 6}$ \\
\hline Intracranial and intraspinal embryonal tumours & $\mathbf{0 . 9 7 3 8}$ & $\mathbf{0 . 9 7 3 8}$ \\
\hline Intracranial and intraspinal germ cell tumours & $\mathbf{0 . 9 8 9 0}$ & 0.9674 \\
\hline Malignant gonadal germ cell tumours & $\mathbf{0 . 9 9 4 1}$ & 0.9530 \\
\hline Nephroblastoma and other nonepithelial renal tumours & $\mathbf{0 . 9 9 7 0}$ & 0.9969 \\
\hline Neuroblastoma and ganglioneuroblastoma & $\mathbf{0 . 9 9 9 8}$ & $\mathbf{0 . 9 9 9 8}$ \\
\hline Retinoblastoma & $\mathbf{1 . 0 0 0}$ & $\mathbf{1 . 0 0 0}$ \\
\hline Average & $\mathbf{0 . 9 8 5 4}$ & 0.9707 \\
\hline
\end{tabular}

2 


\section{Table 3(on next page)}

Estimated carcinogenesis parameters for 10 childhood/young adulthood cancer types.

The parameters are determined for the gamma/Erlang distribution fitted to actual cancer incidence data (see Figure 3). 
1

\begin{tabular}{|l|l|l|l|}
\hline & $\boldsymbol{k}$ & $\boldsymbol{b}$ & $\boldsymbol{A} / \mathbf{1 0 0 0}$ \\
\cline { 2 - 4 } Cancer type & $\begin{array}{l}\text { Number } \\
\text { of } \\
\text { driver } \\
\text { events }\end{array}$ & $\begin{array}{l}\text { Average } \\
\text { time } \\
\text { betwee } \\
\mathbf{n} \\
\text { events, } \\
\text { years }\end{array}$ & $\begin{array}{l}\text { Maximal } \\
\text { populational } \\
\text { susceptibility } \\
\text { \% }\end{array}$ \\
\hline Ewing tumour and related sarcomas of bone & 4.25 & 0.0846 \\
\hline $\begin{array}{l}\text { Extracranial and extragonadal germ cell tumours of } \\
\text { childhood }\end{array}$ & 0.4 & 2.35 & 0.0322 \\
\hline $\begin{array}{l}\text { Extracranial and extragonadal germ cell tumours of } \\
\text { young adulthood }\end{array}$ & 6.5 & 5.3 & 0.111 \\
\hline Hepatoblastoma & 1.55 & 1.4 & 0.0338 \\
\hline Intracranial and intraspinal embryonal tumour & 1.0 & 14.85 & 0.179 \\
\hline Intracranial and intraspinal germ cell tumours & 5.65 & 3.05 & 0.0426 \\
\hline Malignant gonadal germ cell tumours & 8.95 & 3.9 & 1.966 \\
\hline $\begin{array}{l}\text { Nephroblastoma and other nonepithelial renal } \\
\text { tumours }\end{array}$ & 1.75 & 2.2 & 0.124 \\
\hline Neuroblastoma and ganglioneuroblastoma & 1.0 & 2.5 & 0.179 \\
\hline Retinoblastoma & 1.3 & 1.45 & 0.072 \\
\hline
\end{tabular}

2 
Figure 1

Goodness of fit of the gamma/Erlang distribution to the age distributions of incidence of 10 childhood/young adulthood cancer types as a function of various parameter combinations.

The $\boldsymbol{k}$ and $\boldsymbol{b}$ parameters were sampled with 0.05 interval, and for each pair the amplitude ( $\boldsymbol{A}$, not shown) and goodness of fit $\left(R^{2}\right)$ were calculated. See Supplemental Material for the other distributions. 

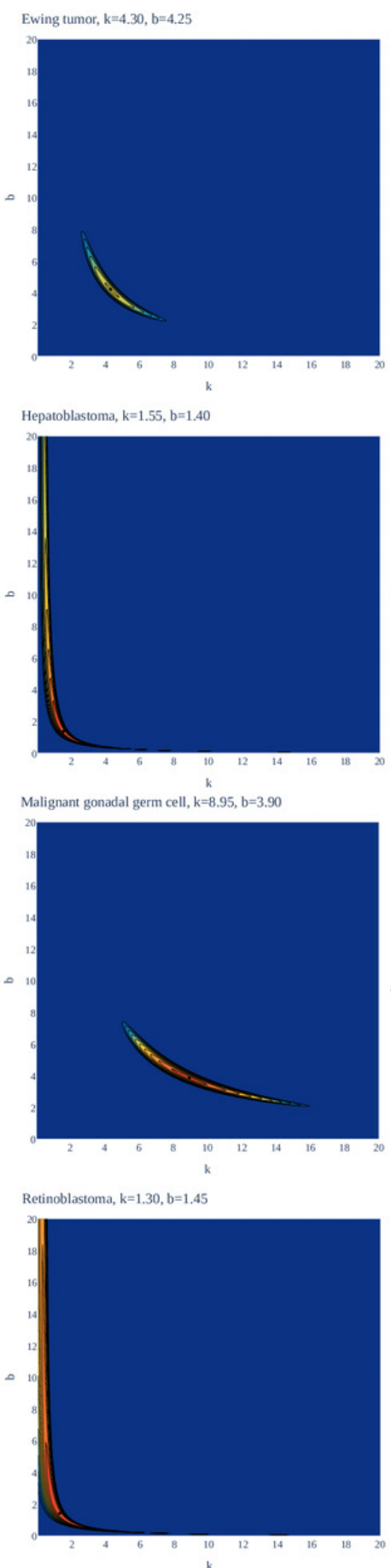
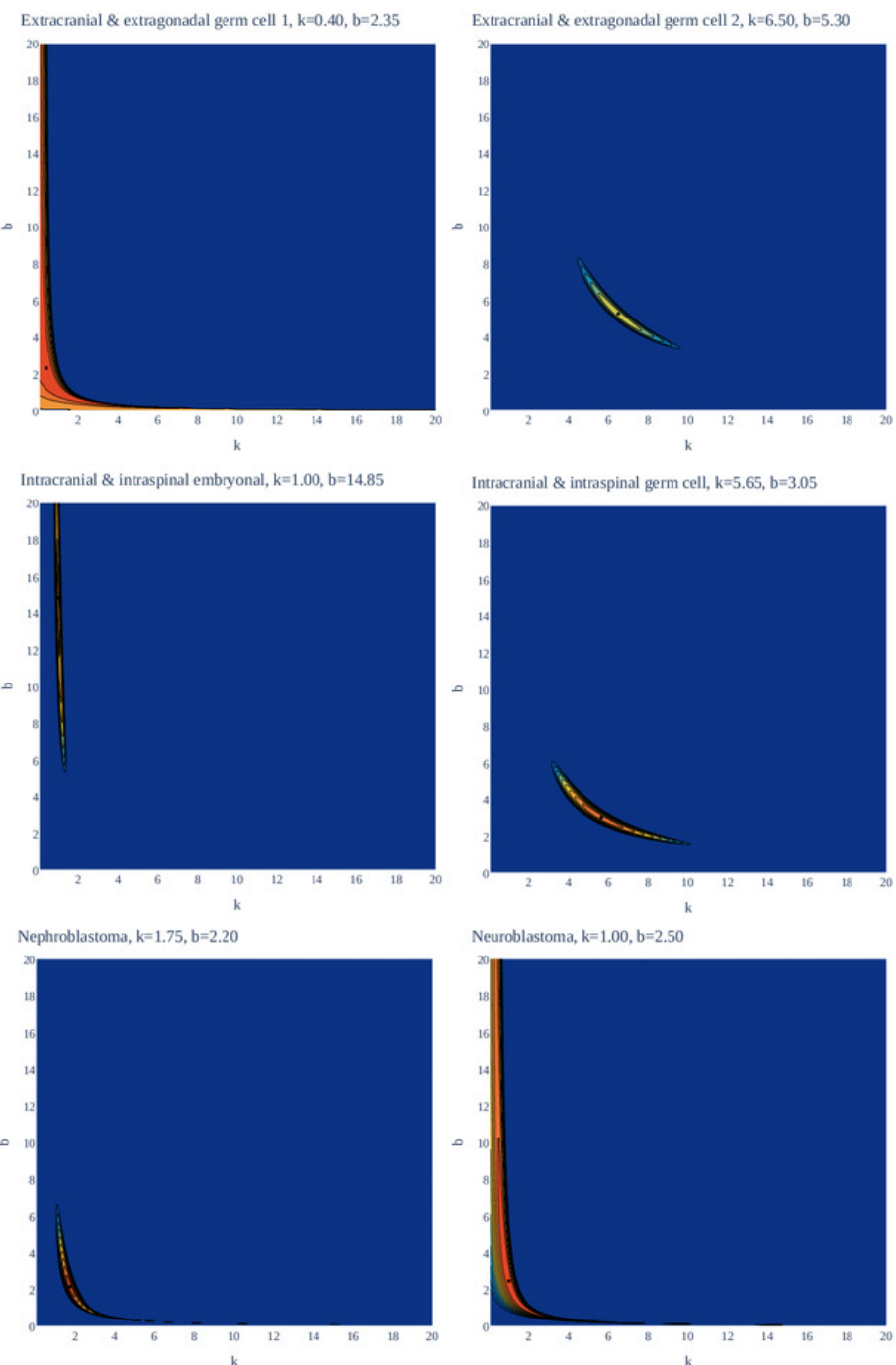

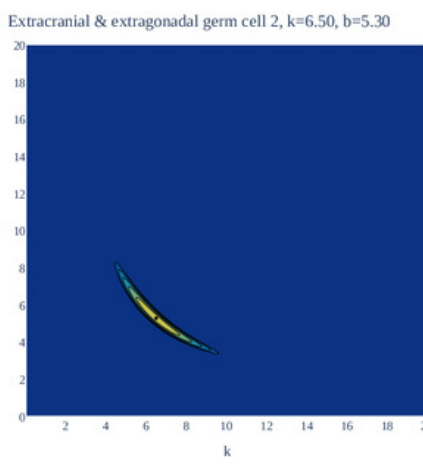

- Optimal

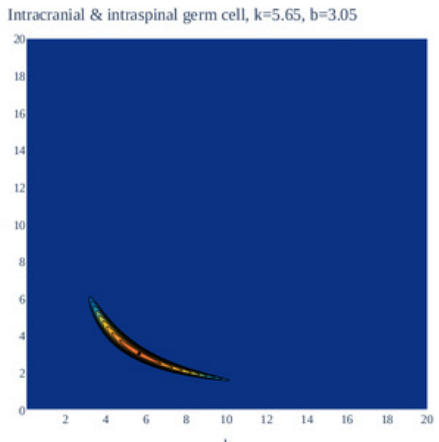

$k$

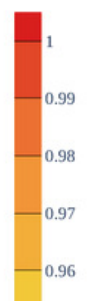

$-0.95 R^{2}$

$-0.94$

$-0.93$

$-0.92$

0.91

0.9 


\section{Figure 2}

Only the gamma/Erlang and the Weibull distributions fit the actual age distributions of childhood and young adulthood cancer incidence without requiring negative age values.

Dots indicate crude incidence rates for 5-year age groups, curves indicate probability density functions fitted to the incidence data for various childhood/young adulthood cancer types. The middle age of each age group is plotted. See Supplemental Material for the optimal parameter values. 

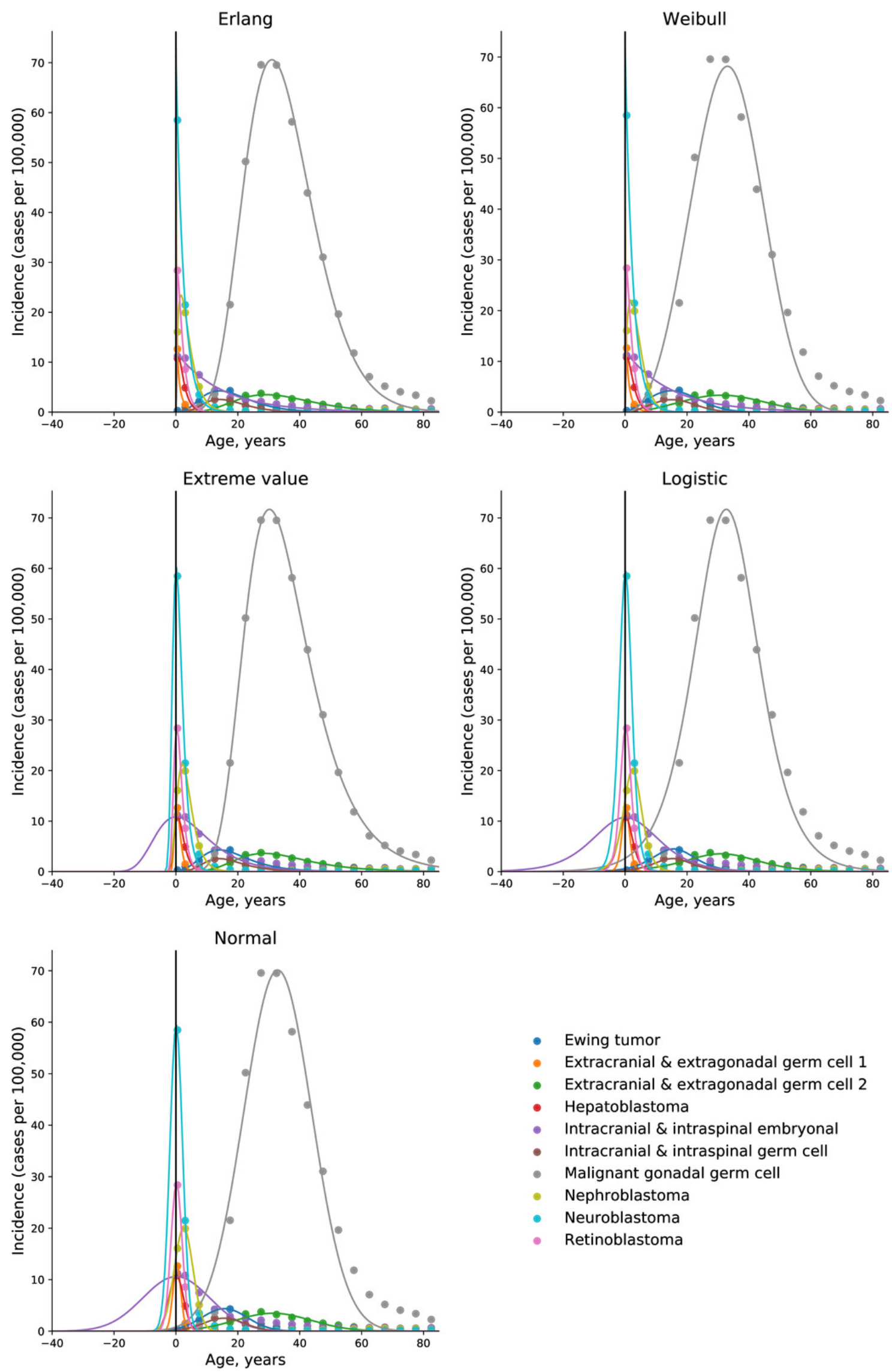

- Ewing tumor

- Extracranial \& extragonadal germ cell 1

- Extracranial \& extragonadal germ cell 2

- Hepatoblastoma

- Intracranial \& intraspinal embryonal

- Intracranial \& intraspinal germ cell

- Malignant gonadal germ cell

- Nephroblastoma

- Neuroblastoma

- Retinoblastoma 


\section{Figure 3}

The gamma/Erlang distribution approximates the age distribution of incidence for childhood and young adulthood cancers better than the Weibull distribution.

Dots indicate crude incidence rates for 5-year age groups, curves indicate the probability density function of the gamma/Erlang (red) or Weibull (blue) distribution fitted to the incidence data (see Table 2 for $\mathrm{R}^{2}$ comparison and Table 3 for estimated parameters). The middle age of each age group is plotted. Extracranial and extragonadal germ cell tumours of childhood and young adulthood are shown on the same plot. 

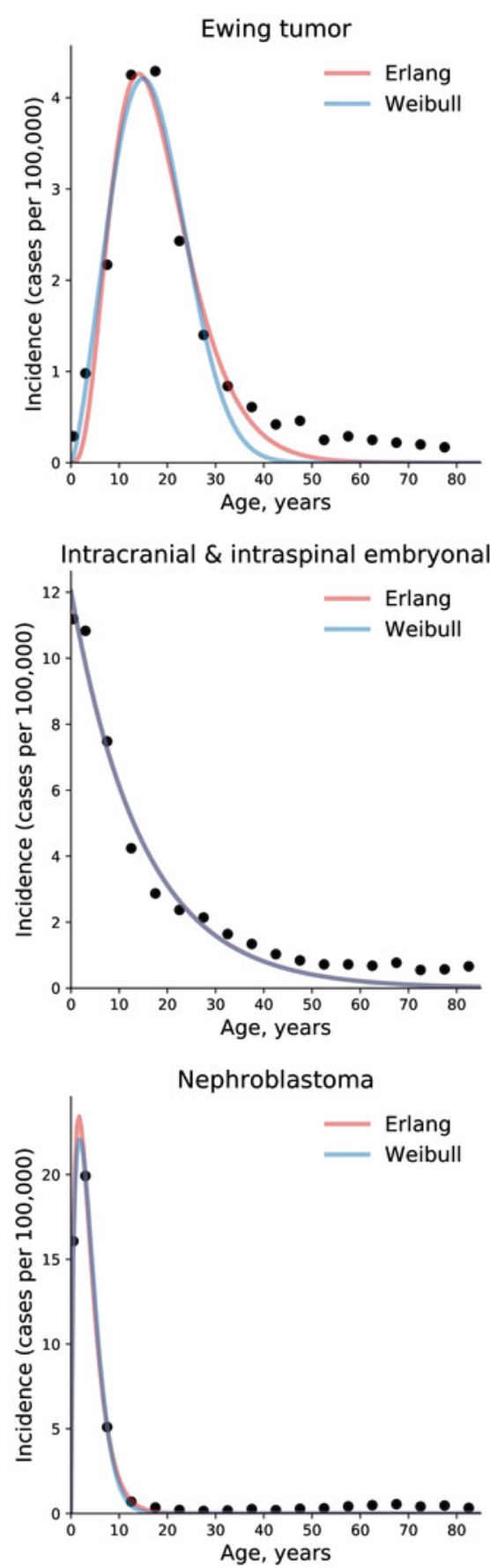
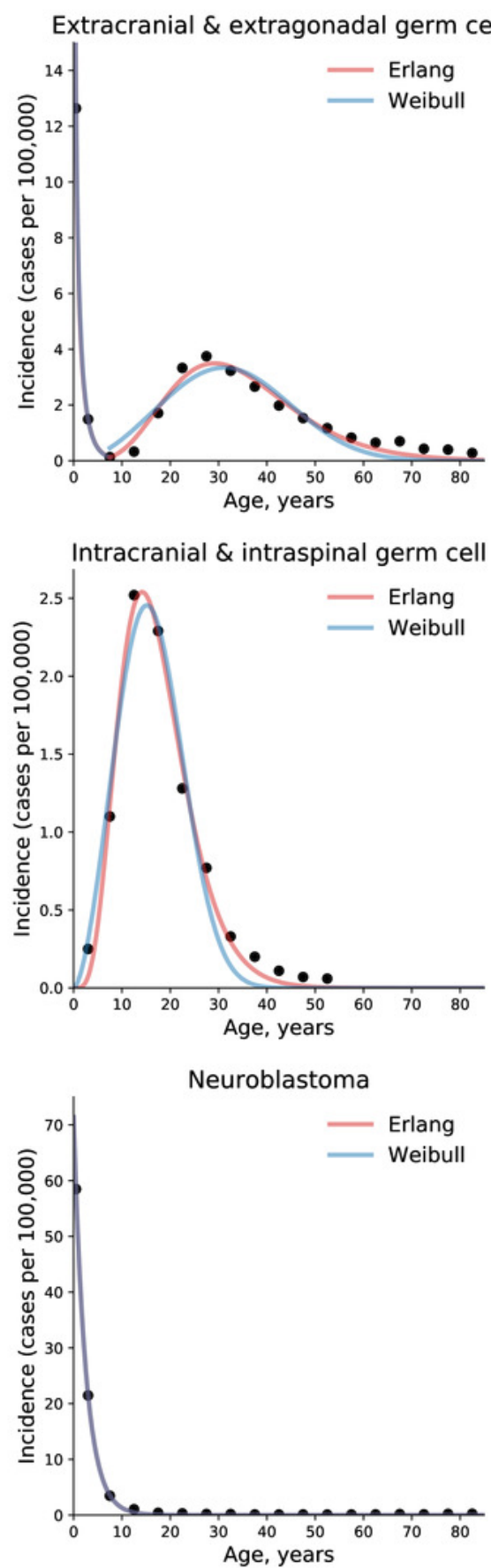
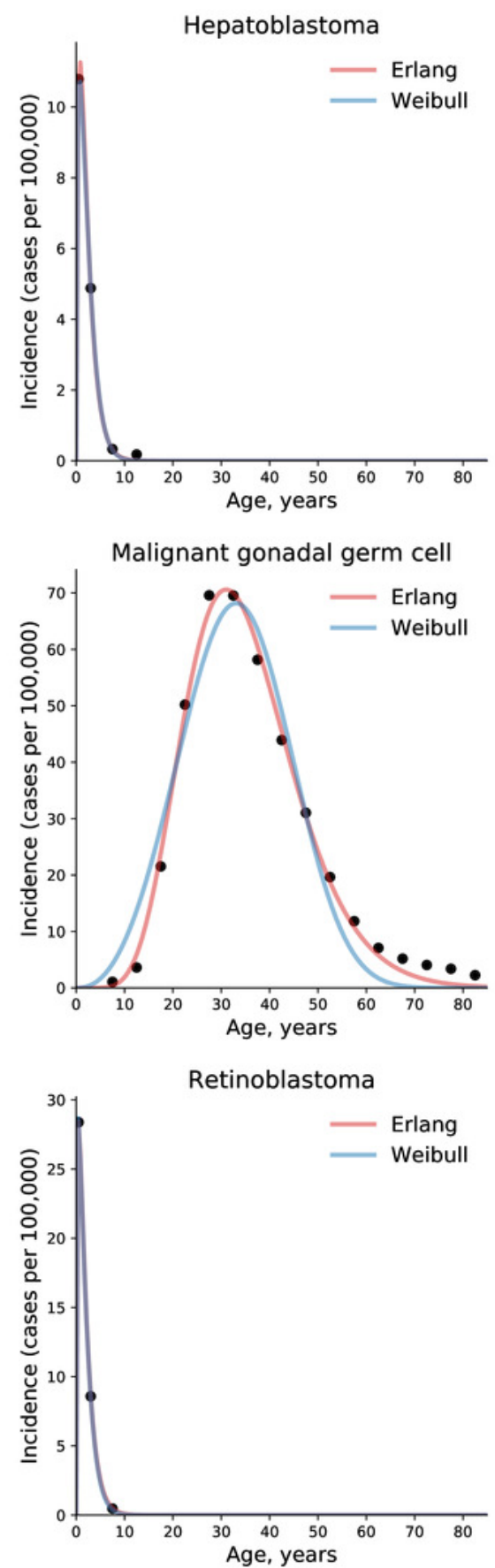